\title{
Clinical and dermoscopic study of melanocytic nevi
}

\section{Bhute Soumya, Bhadbhade P Shweta, Mallikarjun Mallaiah, Kulkarni Sidhant}

\author{
Department of Dermatology, KVG Medical College \& Hospital, Sullia, India
}

Corresponding author: Dr. Bhadbhade P Shweta, E-mail: bhadbhadeshweta5@gmail.com

\begin{abstract}
Background: A dermoscope is a non-invasive, diagnostic tool which visualizes subtle clinical patterns of skin lesions and subsurface skin structures not normally visible to the unaided eye. Thus, dermoscopy has been introduced as an additional measure to make the diagnosis of melanocytic nevi more accurate. Objective: To study clinical and dermoscopic features of Melanocytic nevi. Materials and Methods: A total of 50 patients attending Dermatology OPD in a tertiary care centre with melanocytic nevi during the period from March 2018 to August 2018 were consecutively included in the study. A pre-structured proforma was used to collect baseline data.A detailed history was taken, clinical and dermatological examination done. The Melanocytic nevi were evaluated using digital dermoscope. Results: Out of 50 cases, age distribution was between 16 years and 54 years. The mean age was $33.9 \pm 8.38$ yrs. Male to female ratio was 0.78:1. Face was most common site involved. Among local features Pigment network was the most common pattern seen in all lesions. Pigmentation was regular. Dots and Globules were seen in 52\% of lesions. There were no streaks, bluish white veil, regression structures or hypopigmentation seen. Among global structures reticular network was seen in $76 \%$ of lesions. Globular network was seen in 16\% of lesions. Final impression after clinical and dermoscopic examination, incidence of melanocytic nevi was as follows- junctional nevi as most common $48 \%$, followed by compound nevi $36 \%$ and congenital melanocytic nevi was $16 \%$. Conclusion: Dermoscopy is an evolving science. It serves as a link between macroscopic skin lesions and microscopic histopathological features. Since it is non-invasive, it can be used in all age groups including children and elderly, reducing the need for interventional procedures like skin biopsy. Dermoscopy is a much needed investigative tool in the assessment of Melanocytic nevi.
\end{abstract}

Key words: Dermoscopy; Melanocytic nevi; Pigment network

\section{INTRODUCTION}

A dermoscope (dermatoscope) is a non-invasive, diagnostic tool which visualizes subtle clinical patterns of skin lesions and subsurface skin structures not normally visible to the unaided eye. It has also been called a skin surface microscope, epiluminescence microscope or episcope. Some dermoscopic patterns are observed consistently with certain diseases and these then could be used for their diagnosis. Hence, this office procedure may obviate the need for a skin biopsy for diagnosis and for follow-up. The facility of storage of images and the results being immediately available are added advantages. Basically, a dermoscope is functionally similar to a magnifying lens but with the added features of an inbuilt illuminating system, a higher magnification which can be adjusted, the ability to assess structures as deep as in the reticular dermis, and the ability to record images [1].

The basic principle of dermoscopy is transillumination of a lesion and studying it with a high magnification to visualize subtle features [2]. Light incident on skin undergoes reflection, refraction, diffraction and absorption. These phenomena are influenced by physical properties of the skin [1].

Dermatoscopes are modified magnifying devices that permit the visualization of pigmented structures or vessels in the epidermis and superficial dermis. Because most dermoscopic structures correspond to specific histo-pathologic correlates, dermoscopy can be regarded as a link between clinical (macroscopic) and histopathologic (microscopic) morphology [3]. 
The standard methods to diagnose pigmented skin lesions, such as simple clinical inspection and biopsy, vary in their reproducibility and invasiveness, and there is a need for noninvasive methods that help the clinician in day today practice. The use of dermoscopy improves the diagnostic accuracy and may contribute in discerning the behavior of pigmented skin lesions [4].

The most important parameters that should be assessed when applying dermoscopy in general dermatology include morphological vascular patterns, arrangement of vascular structures, colors, and follicular abnormalities (where relevant) - and the presence of other specific features (clues) [5].

The most studied dermatoscopic method for study of pigmented skin lesion is pattern analysis. Dermatoscopic patterns are mostly derived from the presence or union of 3 main dermatoscopic features: pigment network, globules and homogeneous areas. The pigment network is a grid-like network consisting of pigmented lines and hypopigmented "holes" and represents one of the major dermatoscopic features associated with melanocytic lesions [6].

Dermatoscopyhelps in the diagnosis of many pigmented skin lesions such as seborrheic keratosis (SK), pigmented basal cell carcinoma (BCC), hemangioma, blue nevus, atypical nevus, and cutaneous melanoma. It is $10-27 \%$ more sensitive than clinical criteria of ABCD (asymmetry, border regularity, color distribution, and diameter) in the early diagnosis of cutaneous melanoma. Dermatoscopy of melanocytic lesions increases the presurgical accuracy rate of clinical diagnosis from 50 to $85 \%$ [7].

Undoubtedly, histopathologic examination represents the gold standard of diagnosis in dermatology, and dermoscopy was never suggested as an alternative or competitive method. In contrast, the dermatoscope is a clinical tool that should be considered similar to the stethoscope of general practitioners [8]. Dermoscopy is a valuable tool for evaluating pigmentary lesions, and it greatly enhances the clinical diagnosis of nearly all pigmented skin tumours [9].

The present study has been undertaken to study the clinical and dermoscopic features of melanocytic nevi.

(c) Our Dermatol Online 3.2019

\section{MATERIALS AND METHODS}

\section{Objectives}

To study clinical and dermoscopic features of Melanocytic nevi.

\section{Methodology}

\section{Source of data}

A total of 50 patients attending dermatology attending Out Patient Department of Dermatology, Venereology and Leprosy, KVG Medical College and Hospital, Sullia, with melanocytic nevi during the period from March 2018 To August 2018 were consecutively included in the study after taking informed consent.

\section{Method of Collection of Data}

\section{Study design}

Hospital based cross sectional study.

\section{Sample size}

A total of 50 patients with Melanocytic nevi attending the Out Patient Department of Dermatology, Venereology and Leprosy, KVG Medical college and hospital, Sullia will be consecutively selected for the study.

Sample size was calculated based on a study by Senthikumar et al [10]

Sample size $=4 \mathrm{pq} / \mathrm{d}^{2}$

$\mathrm{p}-$ Prevalence $=0.96 \%$

$\mathrm{q}-100-\mathrm{p}=99.04 \%$

$\mathrm{d}-$ allowable error $=5 \%$

Sample size $=4 \times 0.96 \times 99.04 / 5^{2}$

$$
=380.31 / 25
$$$$
=15.21
$$

As the minimum sample size required for the study is 15.21 , for the present study 50 patients with melanocytic nevi were selected.

\section{Study instrument}

Pre-prepared questionnaire, digital photograph and dermoscope.

\section{Method of collection of data}

1) Patients coming to OPD with Melanocytic nevi will be selected for the study. 
2) The patients will be explained about the nature of the study and written consent of the participating patients and the guardians in case of minors will be taken.

3) A pre-structured proforma shall be used to collect baseline data.

4) Adetailed history is taken, clinical and dermatological examination done.

5) The pigmented skin lesions will be evaluated using digital dermoscope.

6) A skin biopsy and histopathological examination will be done as and when necessary.

\section{Inclusion criteria}

1) Patients with Melanocytic Nevi

\section{Exclusion criteria}

1) Patients who have undergone invasive or non invasive procedure on the lesion during past 6 weeks.

2) Use of depigmenting agent.

\section{Ethics statement}

Ethical committee clearance taken and copy enclosed.

\section{RESULTS}

A total of 50 patients with melanocytic nevi were included in the study. The age of the patients ranged between 16-54 years. The mean age of the study is $33.9 \pm 8.38 \mathrm{yrs}$. Majority of the patients were females. Male to female ratio was 0.78:1 (Table 1). Most of the patients gave chronic history. None of the lesions showed any change in appearance of lesion.

On examination face was the most common site (34\%) followed by the forearm (16\%). Most of the lesions were round in shape (84\%) and majority of the lesions had smooth surface(64\%). Majority of the lesions $(52 \%)$ were of size $6-10 \mathrm{~mm}$, with mean diameter of $7.04 \pm 3 \mathrm{~mm}$.

Out of 50 patients, 11 were diagnosed with junctional nevi. For rest of the lesions, multiple diagnosis were made, because of inconclusive findings.

\section{Dermoscopic Features}

Distribution of local features- Pigment network (Fig. 1) was the most common local feature seen in all 50 lesions. Dots and globules (Fig. 2) were seen in 26 lesions (52\%). Pigmentation was seen to be

\begin{tabular}{lcc} 
Table 1: Age and sex distribution \\
\hline Age group & Male & Female \\
\hline$<20$ & 1 & 1 \\
$21-30$ & 5 & 12 \\
$31-40$ & 13 & 11 \\
$41-50$ & 2 & 2 \\
$>50$ & 1 & 2 \\
TOTAL & 22 & 28 \\
\hline
\end{tabular}

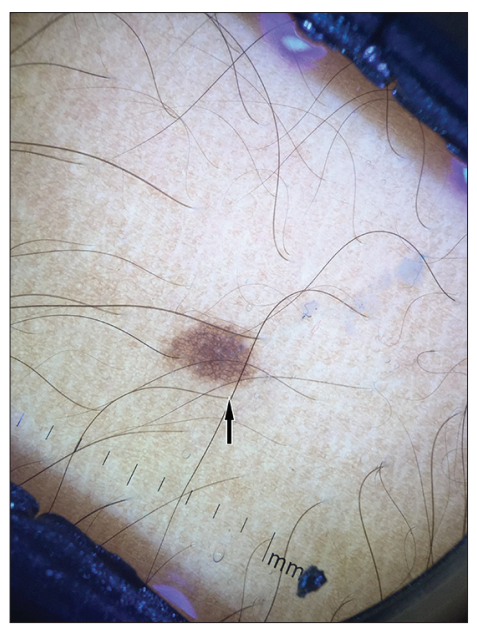

Figure 1: Reticular pigment network.

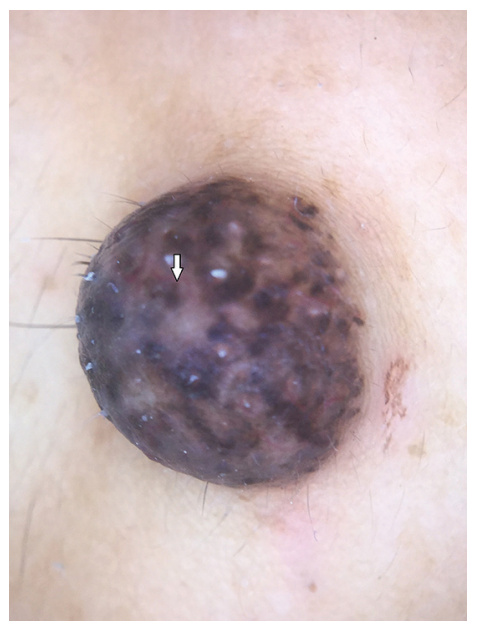

Figure 2: Dots and globules and few hair follicles.

regular in all lesions (Table 2). Distribution of global structures-globular network was seen in 42 lesions and reticular network was seen in 38 lesions (Table3).

Final impression after clinical and dermoscopic examination, incidence of melanocytic nevi was as follows- junctional nevi with reticular network (Fig. 1) as most common $48 \%$, followed by compound nevi with dots and glocules and few hair follicles (Fig. 2) 36\% and congenital melanocytic nevi with fine reticular network with perifollicular hyperpigmentation (Fig. 3) was $16 \%$ (Table 4). 
Table 2: Local features

\begin{tabular}{lcc}
\hline Local features & Frequency & Percentage \\
\hline Pigment network & 50 & 100 \\
Dots \& globules & 21 & 42 \\
Streaks & 0 & 0 \\
Bluish white veil & 0 & 0 \\
Hypopigmentation & 0 & 0 \\
Regression structures & 0 & 0 \\
Vascular structures & 0 & 0 \\
\hline
\end{tabular}

Table 3: Global features

\begin{tabular}{lcc}
\hline Global features & Frequency & Percentage \\
\hline Reticular network & 38 & 76 \\
Globular network & 42 & 84 \\
Cobble stone pattern & 0 & 0 \\
Homogenous pattern & 0 & 0 \\
Starburst pattern & 0 & 0 \\
Parallel pattern & 0 & 0 \\
Multi cimponent pattern & 0 & 0 \\
Unspecific pattern & 0 & 0 \\
\hline
\end{tabular}

Table 4: Final impression

\begin{tabular}{ccc}
\hline Final impression & Frequency & Percentage \\
\hline Congenital Melanocytic Nevi & 8 & 16 \\
Compound Nevus & 18 & 36 \\
Junctional Nevi & 24 & 48 \\
\hline
\end{tabular}

\section{DISCUSSION}

Nevi are common benign proliferations of uniform melanocytes. Although 20-30\% of melanomas arise in association with pre-existing naevi, malignant transformation of naevi is a very rare event [11]. A randomized clinical trial of dermatologists trained in dermoscopy revealed a $42 \%$ reduction in the number of unnecessary biopsies compared with using the naked eye alone [12]. Hence regular dermoscopic examination of melanocytic nevi is important for early detection of melanoma.

The most commonly involved age group in this study was $31-40$ years with a mean age of 33.9 years $+/-8.38$. A study by Piazza et al., mean age was $8.4 \pm 3.5$ years [13]. A study of dermoscopic patterns of congenital melanocytic nevi by Changchien et al., showed a mean age of 35.2 years [14]. In another study of dermoscopic patterns of congenital melanocytic nevi by Cengiz et al., out of 108 patients Sixty-two participants $(57.4 \%)$ were aged less than 16 years, and 46 participants $(42.6 \%)$ were aged 16 and more [15].

In this study there was female preponderance. Male to female ratio was $0.78: 1$. A study by Piazza et al., both male and females were equally affected [13]. In

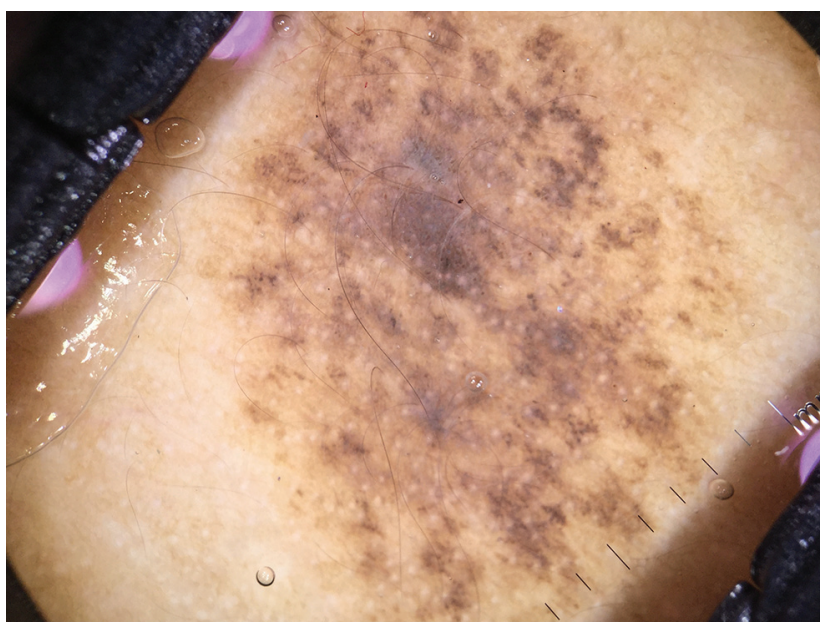

Figure 3: Fine reticular pigment network and few perifollicular hyperpigmentation.

a study by Changchien et al., out of a study population of 77 individuals, there were 36 females $(47 \%)$ and 41 males (53\%) with male preponderance [14]. According to a study by Cengiz et al., there was similar female preponderance with 57 females out of 108 and 51 males [15].

On examination face was the most common site $(34 \%)$ and next was the forearm(16\%). In a study by Piazza et al., $40 \%$ of lesions were on extremities followed by $37 \%$ on trunk [13]. A study by Changchein et al., trunk was the most common site in $59.3 \%$ of patients followed by extremities [14]. Another study by Cengiz et al., 52\% of lesions were on extremities followed by trunk with $40 \%$ of lesions [15].

Out of 50, 8 lesions were present at birth. According to a study by Piazza et al., 5 out of 201 lesions were present at birth. Mean diameter of the lesions was $7.04 \pm 3 \mathrm{~mm}$. A study by Piaza et al., the mean diameter of the lesions was $2.3 \pm 1.4 \mathrm{~mm}$ [13]. In this study $84 \%$ of the lesions were round followed by $16 \%$ lesions were round to oval. In a study by Changchien et al., 79\% of lesions were round in shape followed by irregular shape in $14 \%$ [14].

On dermoscopic examination, pigment network was seen in all $100 \%$ of lesions, dots and globules were noted in $42 \%$, reticular network was seen in $76 \%$ and globular network in $84 \%$ of lesions. In a study by Piazza et al., the most prevalent dermoscopic structures observed in the lesions were dots (72.6\%), followed by structureless areas $(47.8 \%)$, pigment network $(40.8 \%)$ and globules (28.4\%). The presence of branched streaks was observed in only $5.5 \%$ of the lesions [13]. In a study by Cengiz et 
al., after dermoscopy it was found that reticular pattern was in $24.1 \%$, globular pattern in $32.4 \%$, reticularglobular pattern in $12 \%$, homogeneous pattern in $14.8 \%$, reticularhomogeneous pattern in $5.6 \%$, globularhomogeneous pattern in $1.9 \%$, cobblestone pattern in $6.5 \%$, reticular patchy pattern in $2.8 \%$. The globular pattern as the predominant dermoscopic pattern was more frequent in children younger than 16 years old $(32.4 \%)$ [15].

As presented, our observations were partially comparable with the findings of previous studies. The aim of this work was to evaluate the dermoscopic structures and patterns of Melanocytic nevi.

\section{CONCLUSION}

In our study, pigment network was seen in all $100 \%$ of lesions, globular pattern was observed in $84 \%$ and dots and globules in $42 \%$. Dermoscopy is an evolving science. It serves as a link between macroscopic skin lesions and microscopic histopathological features. Since it is non-invasive, it can be used in all age groups including children and elderly, reducing the need for interventional procedures like skin biopsy. Dermoscopy is a much needed investigative tool in the assessment of Melanocytic nevi.

\section{Statement of Human and Animal Rights}

All procedures followed were in accordance with the ethical standards of the responsible committee on human experimentation (institutional and national) and with the Helsinki Declaration of 1975, as revised in 2008.

\section{Statement of Informed Consent}

Informed consent was obtained from all patients for being included in the study.

\section{REFERENCES}

1. Nischal KC, Khopkar U. Dermoscope. Indian J Dermatol Venereol Leprol. 2005;71:300-3.

2. Stolz W, Bilek P, Landchaer M, Amandcogneta. Basis of dermatoscopy and skin-surface microscopy. William Stolz, Peter Bilek, Michael Landchaer, Amandcogneta.In: Color atlas of dermatoscopy. $1^{\text {st }}$ ed. Germany: Blackwell Publications; 1994:7-10.

3. Zalaudek I, Kreusch J, Giacomel J, Ferrara G, Catricalà C, Argenziano G. How to diagnose nonpigmented skin tumors: A review of vascular structures seen with dermoscopy. J Am Acad Dermatol. 2010;63:361-74.

4. Dogra S, Mittal A. Role of dermoscopy in the diagnosis of pigmentary dermatoses in skin of color. Pigment Int. 2014;1:41-3.

5. Panasiti V, Devirgiliis V, Curzio M, Roberti V, Gobbi S, Masciangelo $\mathrm{R}$, et al. The reticular point of view in dermatoscopy. J Am Acad Dermatol. 2009;61:605-10.

6. Senel E. Dermatoscopy of non-melanocytic skin tumors. Indian J Dermatol Venereol Leprol 2011;77:16-22.

7. Lallas A, Argenziano G. Dermatoscope-the dermatologist's stethoscope. Indian J Dermatol Venereol Leprol. 2014;80:493-4.

8. Kim G-W, Jung H-J, Ko H-C, Kim M-B, Lee W-J, Lee S-J, et al. Dermoscopy can be useful in differentiating scalp psoriasis from seborrhoeic dermatitis. Br J of Dermatol. 2010;164:652-6.

9. Senthikumar M, Thappa DM. Melanocytic nevi in children: A clinical study. Indian J Dermatol. 2006;51:26-9.

10. Stefanaki I, Antoniou C, Stratigos A. Benign melanocytic proliferations and melanocytic naevi. In: Griffiths C, Barker J, Bleiker T, Chalmers R, Creamer D, editors. Rook's textbook of dermatology $9^{\text {th }}$ ed. Oxford: Blackwell publishing; 2016;132:1-47.

11. Carli P, de Giorgi V, Chiarugi A, Nardini P, Weinstock MA, Crocetti E, et al. Addittion of dermoscopy to conventional nakedeye examination in melanoma screening: a randomized study. J Am Acad Dermatol. 2004;50:683-9.

12. Piazza CD, Yamada S, Marcassi AP, Maciel MG, Seize MP, Cestari SP. Dermoscopic patterns of melanocytic nevi in children and adolescents: a cross-sectional study. An Bras Dermatol. 2017;92:340-4.

13. Changchien L, Dusza SW, Agero AL, Korzenko AJ, Braun RP, Sachs D, et al. Age- and site-specific variation in the dermoscopic patterns of congenital melanocytic nevi: an aid to accurate classification and assessment of melanocytic nevi. Arch Dermatol. 2007;143:1007-14.

14. Cengiz FP, Emiroglu N, Ozkaya DB, Su O, Onsun N. Dermoscopic Features of Small, Medium, and Large-Sized Congenital Melanocytic Nevi. Ann Dermatol. 2017;29 26-32.

Copyright by Bhute Soumya, et al. This is an open-access article distributed under the terms of the Creative Commons Attribution License, which permits unrestricted use, distribution, and reproduction in any medium, provided the original author and source are credited.

Source of Support: Nil, Conflict of Interest: None declared. 